\title{
ON THE BEHAVIOUR OF ANALYTIC FUNCTIONS IN THE NEIGHBOURHOOD OF THE BOUNDARY OF A RIEMANN SURFACE
}

\section{A. CORNEA}

1. Mr. Z. Kuramochi has recently obtained the following very interesting result concerning the classification of Riemann surfaces [1]:

If we delete from a Riemann surface belonging to the class $O_{H B}-O_{G}\left(O_{H D}-O_{G}\right)$ an arbitrary compact set, the resulting surface remains in the class $O_{A B}\left(O_{A D}\right)$.

In the present paper we indicate a new method of proving this theorem, simultaneously for the cases $B$ and $D$.

Let $R$ be a non-compact Riemann surface, $\beta$ its ideal boundary and $\alpha$ a closed curve, dividing $R$ into the domains $R^{\prime}$ and $R^{\prime \prime}$; suppose that $R^{\prime}$ is noncompact and let $\beta^{\prime}$ be the part of the ideal boundary $R$ which belongs to $R^{\prime}$. We shall call $\beta^{\prime}$ a boundary part and $R^{\prime}$ a neighbourhood of $\beta^{\prime}$.

Definition. A boundary part $\beta^{\prime}$ is a $O_{H B}\left(O_{H D}\right)$-boundary part if for each completion of $R^{\prime}$ obtained by adding along $\alpha$ a compact domain, the resulting surface belongs to the class $O_{H B}-O_{G}\left(O_{H D}-O_{G}\right)$.

L. Sario proved the following necessary and sufficient condition satisfied by a surface which belongs to $O_{H B}\left(O_{H D}\right)$ [2]:

Let $R$ be a Riemann surface and $\alpha$ a finite system of closed curves, bounding a compact domain $K$ on $R$. Then, $R$ belongs to $O_{H B}\left(O_{H D}\right)$ if and only if each bounded (Dirichlet bounded ${ }^{* \prime}$ ) harmonic function in $R-K$, which is zero on $\alpha$ and whose conjugate has on $\alpha$ a zero period, vanishes in $R-K$ identically.

This criterion shows that in the definition of a $O_{H B}\left(O_{H D}\right)$-boundary part, it is enough that at least one completion of $R^{\prime}$ by adding a compact set along $\alpha$ belongs to $O_{H D}-O_{G}\left(O_{H D}-O_{G}\right)$.

Consider now a non-compact Riemann surface $R$; let $\alpha$ be an analytic

Received April 3, 1957.

"I.e., with a finite Dirichlet integral. 
curve, dividing $R$ and $R^{\prime}$ one of the non-compact components of $R-\alpha$. Let $\left\{R_{n}\right\}$ be an exhaustion of $R, \beta_{n}$ the boundary of $R_{n}, R_{n}^{\prime}=R_{n} \cap R^{\prime}, \beta_{n}^{\prime}=\beta_{n} \cap R^{\prime}$; and let $u$ be a function, harmonic in a neighbourhood of $\alpha$. Let

$$
H(u)=\lim _{n \rightarrow \infty} H_{n}(u),
$$

where $H_{n}(u)$ is the harmonic function in $R_{n}^{\prime}$ which coincides with $u$ on $\alpha$ and is zero on $\beta_{n}^{\prime}$ [3], [4]. The convergence of the sequence $H_{n}(u)$ is uniform on every compact set belonging to $R^{\prime}$ and the limit is bounded and has a finite Dirichlet integral in $R^{\prime} . H(u)$ is a linear operator.

2. Lemma. If $R^{\prime}$ is a neighbourhood of an $O_{H B}\left(O_{H D}\right)$-boundary part $\beta^{\prime}$ of a Riemann surface $R$, and if the relative boundary $\alpha$ of $R^{\prime}$ is analytic, then every bounded (Dirichlet bounded) harmonic function in $R^{\prime}$ satisfies the equality

$$
u-a=H(u-a)
$$

with a suitable constant $a$.

Indeed, the function

$$
u-a-H(u-a)
$$

is harmonic and bounded (Dirichlet bounded) in $R^{\prime}$ and zero on $\alpha$. We can always determine the constant $a$ in such a way that the conjugate of $u-a-H(u-a)$ has a zero period on $\alpha$. The lemma follows then from Sario's criterion, stated above.

Denoting by $\omega$ the harmonic measure of $\beta^{\prime}$ with respect to $R^{\prime}$, i.e.,

$$
\omega=\lim _{n \rightarrow \infty} \omega_{n},
$$

where $\omega_{n}$ is harmonic in $R_{n}^{\prime}$ and takes constant values 0 on $\alpha$ and 1 on $\beta_{n}^{\prime}$, we have

$$
\begin{aligned}
|u-a|=|H(u-a)| & =\lim _{n \rightarrow \infty}\left|H_{n}(u-a)\right| \\
& \leqq \lim _{n \rightarrow \infty} M\left(1-\omega_{n}\right)=M(1-\omega),
\end{aligned}
$$

where $M=\max |u-a|$ on $\alpha$. This inequality implies that $u$ converges to the constant $a$ on every sequence of points on which $\omega$ converges to 1 .

3. Theorem. A Riemann surface $R$ which contains in its boundary $a$ $O_{I I B}\left(O_{H D}\right)$-boundary part, belongs to the class $O_{A B}\left(O_{A D}\right)$. 
Let $R^{\prime}$ be a neighbourhood of $\beta^{\prime}$ with an analytic curve $\alpha$ as relative boundary. Consider, as above, an exhaustion $\left\{R_{n}\right\}$ of $R$ and let $\beta_{n}$ be the boundary 'of $R_{n}, \quad R_{n}^{\prime}=R_{n} \cap R^{\prime}$ and $\beta_{n}^{\prime}=\beta_{n} \cap R^{\prime}, \omega=\lim \omega_{n}$ be the harmonic measure of $\beta^{\prime}$ with respect to $R^{\prime}$. Denote by $G_{\varepsilon}$ the set of points in which $\omega<1-\varepsilon(0<\varepsilon<1)$ and let $\beta_{\varepsilon}$ be the level set $\omega=1-\varepsilon$.

Let $w=u+i v$ be a bounded (Dirichlet bounded) analytic function on $R$. The lemma implies the existence of two constants $a$ and $b$ such that on $R^{\prime}$

$$
\begin{aligned}
& u-a=H(u-a) \\
& v-b=H(v-b) .
\end{aligned}
$$

Setting $c=a+i b$, we get

$$
|w-c| \leqq|u-a|+|v-b| \leqq 2 M(1-\omega)
$$

where $M$ is the maximum of $|w-c|$ on $\alpha$. The following inequality holds in $G_{\varepsilon} \cap R_{n}^{\prime}$

$$
\log \left|\frac{w-c}{2 M}\right| \leqq\left(\frac{\omega}{1-\varepsilon}-\omega_{n}^{\prime}\right) \log \varepsilon
$$

where $\omega_{n}^{\prime}$ is the bounded harmonic function in $R_{n}^{\prime} \cap G_{s}$ which is 0 on $\alpha$ and on $\beta_{\varepsilon} \cap R_{n}^{\prime}$ and 1 on $\beta_{n}^{\prime} \cap G_{\varepsilon}$. Indeed, this inequality is obviously satisfied on the boundary of $R_{n}^{\prime} \cap G_{\varepsilon}$. We have in $G_{\varepsilon} \cap R_{n}^{\prime}$

$$
\omega_{n}^{\prime} \leqq \omega_{n}
$$

and, therefore, in $G_{\varepsilon}$

$$
\omega^{\prime}=\lim _{n \rightarrow \infty} \omega_{n}^{\prime} \leqq \lim _{n \rightarrow \infty} \omega_{n}=\omega \leqq 1-\varepsilon .
$$

If $\omega^{\prime}>0$, then

$$
\sup _{G_{\varepsilon}} \omega^{\prime}=1
$$

which contradicts $(9) .{ }^{*}$ We infer that $\omega^{\prime} \equiv 0$. For $n \rightarrow \infty$, the inequality (7) yields

$$
\log \left|\frac{w-c}{2 M}\right| \leqq \frac{\omega}{1-\varepsilon} \log \varepsilon
$$

in $G_{\varepsilon}$. But $\varepsilon$ is arbitrary and $\omega$ is positive, so that we get

*) Indeed, let $\theta^{\prime}=\sup _{\sigma_{\varepsilon}} \omega^{\prime} \leqq 1$ on $G \varepsilon \cap R_{n}^{\prime}$. Then we have $\frac{\omega^{\prime}}{\theta^{\prime}} \leqq \omega_{n}^{\prime}$; passing to the limit, we get $\frac{\omega^{\prime}}{\theta^{\prime}} \leqq \omega^{\prime}$, i.e., $1 \leqq \theta^{\prime}$. 


$$
w-c \equiv 0
$$

and this proves the theorem.

This theorem gives as an immediate consequence Kuramochi's theorem, conversely, our theorem follows easily from Kuramochi's one.

The following generalization of Kuramochi's theorem for the case $O_{H B}$ was pointed out to the author by C. Constantinescu.

THeOREM. Let $R$ be a Riemann surface belonging to $O_{H B}-O_{G}$ and let $F \subset R$ be a closed but not necessarily compact set, such that the harmonic measure $\omega$ of the ideal boundary of $R$ with respect to $R-F$ be positive. Under these conditions, there exist at least a compoment of $R-F$ on which each analytic function takes all the values with the possible exception of a set of capacity zero.

A proof can be given by the method of $Z$. Kuramochi for the case $O_{H B}$, applied to the component of $R-F$ in which $\omega$ is positive.

\section{BIBLIOGRAPHY}

[1] Kuramochi, Z., On the behaviour of analytic functions on abstract Riemann surfaces, Osaka Math. J., vol. 7, nr. 1 (1955), pp. 109-127.

[2] Sario, L., A linear operator method on arbitrary Riemann surfaces, Trans. Amer. Math. Soc., vol. 72 (1952), pp. 281-295.

[3] Nevanlinna, R., Uniformisierung, Springer Verlag, Berlin (1953), pp. 320-328.

[4] Parreau, M., Sur les moyennes des fonctions harmoniques et analytiques et la classification des surfaces de Riemann, Ann. Inst. Fourier, vol. 3 (1952), pp. 103-197.

Institute of Mathematics

Academy R.P.R.

Bucarest 